\title{
Quantitative detection of the rice false smut pathogen Ustilaginoidea virens by real-time PCR
}

\author{
H. Li ${ }^{1,2,3 *}$, D.H. Ni ${ }^{1,2 *}$, Y.B. Duan ${ }^{2,4}$, Y. Chen ${ }^{5}$, J. Li ${ }^{1,2}$, F.S. Song ${ }^{2,4}$, \\ L. $\mathrm{Li}^{2}$, P.C. Wei ${ }^{2,3}$ and J.B. Yang ${ }^{2}$
}

${ }^{1}$ Key Laboratory of Ion Beam Bioengineering, Institute of Technical Biology and Agriculture Engineering, Chinese Academy of Sciences, Hefei, Anhui, China

${ }^{2}$ Key Laboratory of Rice Genetics Breeding of Anhui Province, Rice Research Institute, Anhui Academy of Agricultural Sciences, Hefei, Anhui, China

${ }^{3}$ Institute of Agricultural Engineering, Anhui Academy of Agricultural Sciences, Hefei, Anhui, China

${ }^{4}$ College of Life Sciences, Anhui Agricultural University, Hefei, Anhui, China ${ }^{5}$ Institute of Plant Protection, Anhui Academy of Agricultural Sciences,

Hefei, Anhui, China

*These authors contributed equally to this study.

Corresponding author: J.B. Yang

E-mail: yjianbo@263.net

Genet. Mol. Res. 12 (4): 6433-6441 (2013)

Received April 25, 2013

Accepted September 15, 2013

Published December 10, 2013

DOI http://dx.doi.org/10.4238/2013.December.10.4

\begin{abstract}
Rice false smut (RFS) is an important rice disease that is caused by the pathogen Ustilaginoidea virens. In this study, we developed a real-time polymerase chain reaction (PCR) assay to detect $U$. virens and to estimate the level of disease. The genomic DNAs of $U$. virens and rice were extracted together from the rice samples. Real-time PCR assays were performed and compared to conventional nested-PCR assays. The realtime PCR assay presented a consistent linearity of the standard curve $\left(\mathrm{R}^{2}=\right.$ 0.9999). The detection limit could be as low as $40 \mathrm{fg} U$. virens DNA with a rice genomic DNA background on using the real-time PCR assay, which
\end{abstract}


showed significantly higher sensitivity than the conventional nested-PCR assay. We conclude that the real-time PCR quantitative assay is a useful tool for detecting $U$. virens and for early defense and control of RFS.

Key words: Rice false smut; Real-time PCR; Detection; Quantification; Ustilaginoidea virens

\section{INTRODUCTION}

Rice (Oryza sativa L.) is the staple food for half of the world's population, especially in Asian countries. Rice false smut (RFS), which is caused by Ustilaginoidea virens (Cke.) Tak., is one of the most common and serious diseases in rice-growing areas of the world $(\mathrm{Ou}$, 1972; Tanaka and Tanaka, 2008). The disease was first reported in Tirunelveli District of Tamil Nadu State of India in 1878 (Ou, 1972). The RFS disease symptoms appear on the spikelets at maturity. The diseased spikelets, the so-called green balls, are covered with powdery darkgreen chlamydospores (Ou, 1972). Outbreaks of the disease could lead to yield loss and reduced grain quality (Lu et al., 2009). In addition, ustiloxins derived from false smut balls are toxic to animals and are a potential source of food contamination (Koiso et al., 1994).

RFS had been considered to be a minor disease because of its infrequent occurrence. However, in recent years, it has become a serious grain disease because of the apparent change in climate, widespread cultivation of high nitrogen fertilizer-responsive varieties, and excessive application of nitrogen. In China, RFS has become a serious fungal disease on rice since the 1980s because japonica rice varieties are widely cultivated, most of which are very susceptible to RFS and are high nitrogen fertilizer-responsive cultivars (Ji and Zheng, 1995). There have been many reports of severe outbreaks of RFS since 2001 in many rice-growing provinces of China, such as Liaoning, Hubei, Sichuan, and Anhui; the yield loss ranged from 20 to 50\% in different areas and varied with rice varieties. In 2005, the occurrence area of RFS disease was approximately 330,000 ha and a third of the panicles in Sichuan were affected (Lu et al., 2009).

Isolation on nutrient-rich media and morphological examinations are the conventional detection and identification methods for $U$. virens (Zhou et al., 2003). However, these procedures are time consuming and nonspecific. Conventional polymerase chain reaction (PCR) and nested-PCR techniques can qualitatively detect $U$. virens. Because of the use of two pairs of amplification primers, the nested-PCR assay has higher sensitivity and specificity than the conventional single-round PCR assay (Zhou et al., 2003). However, it is difficult to quantify the amount of DNA by the conventional PCR or nested-PCR assay. It is necessary to quantify the amount of pathogen DNA when quantitative data about pathogen severity are required. Further, gel electrophoresis, which is the normal tool for analyzing conventional PCR and nested-PCR results, could lead to the detection of contamination or false-positive or falsenegative results (Harrison et al., 2002). In contrast, the widely used real-time PCR method has better quantitative performance, greater sensitivity and greater accuracy (Maciel et al., 2011). Real-time PCR can monitor the progression of DNA amplification after each cycle through fluorescence probes (Ginzinger, 2002). In addition, the chances of contamination are obviously reduced compared to that in conventional PCR because amplification and detection occur in a single sealed tube (Nejat et al., 2010). The TaqMan probe was widely used in real-time PCR because it had better sensitivity and reliability than SYBR Green I, a commonly used fluores- 
cent DNA-binding dye (Nejat et al., 2010). By using the TaqMan probe to detect the ribosomal DNA (rDNA) of $U$. virens, a linear correlation between cycle threshold $\left(\mathrm{C}_{\mathrm{T}}\right)$ values and the number of false smut balls in the soil was revealed (Ashizawa et al., 2010). Therefore, realtime PCR can be used to detect and quantify $U$. virens. However, little information is available about the relationship between the amount of $U$. virens DNA and the level of RFS disease.

For rapid and early detection of $U$. virens and quantification the $U$. virens DNA level, a quantitative detection method is urgently needed. Therefore, the aim of this study was to develop a sensitive and reproducible real-time PCR assay to detect, quantify the $U$. virens, and evaluate the level of disease in rice.

\section{MATERIAL AND METHODS}

\section{Isolation and culture of $U$. virens and rice plants}

An isolate (Uv-A12) of $U$. virens was obtained from a false smut ball produced on a floret of rice that was planted in a paddy field in Hefei, Anhui Province, China. Preparation of the chlamydospore suspension, hyphal culture, and storage were performed according to the method reported by Ashizawa et al. (2010).

To obtain artificially infected rice plants, a conidial suspension $\left(1 \times 10^{6}\right.$ conidia $\left./ \mathrm{mL}\right)$ was injected into the flag leaf sheath of a highly susceptible rice cultivar, Wanjing97 (Oryza sativa $\mathrm{cv}$. Wanjing97), 10 days before heading at the booting stage. From the time of injection to the maturation of rice seeds, rice spikelets were harvested every 10 days. To obtain rice plants in the field, Wanjing97 seedlings were planted in Jinzhai, Anhui Province (RFS outbreak area); Hefei, Anhui Province (RFS epidemic area); and Sanya, Hainan Province (RFS non-epidemic area). All the seeds were harvested and mixed as the detection samples.

\section{DNA extraction}

For $U$. virens or rice DNA extraction, $50 \mathrm{mg}$ frozen mycelia samples of $U$. virens or $50 \mathrm{mg}$ rice spikelet powder of every sample were placed in $1.5-\mathrm{mL}$ microcentrifuge tubes. The DNA extraction procedure has been described in a previous report ( $\mathrm{Li}$ et al., 2013). Briefly, the sample was suspended in $300 \mu \mathrm{L}$ extraction buffer (100 mM Tris-HCl, pH 8.0; $50 \mathrm{mM}$ ethylenediaminetetraacetic acid (EDTA), $\mathrm{pH} 8.0 ; 1 \mathrm{M} \mathrm{NaCl} ; 1.25 \%$ sodium dodecyl sulfate; and 1\% 2-mercaptoethanol) and vigorously vortexed for $1 \mathrm{~min}$. Next, $150 \mu \mathrm{L} 5 \mathrm{M}$ potassium acetate was added to the tube, and the tube was vigorously vortexed again for $1 \mathrm{~min}$. The suspension was centrifuged for $10 \mathrm{~min}$ at $12,000 \mathrm{~g}$. Then, $300 \mu \mathrm{L}$ supernatant was transferred to a new tube with $200 \mu \mathrm{L}$ isopropanol. The suspension was centrifuged for $10 \mathrm{~min}$ at $12,000 \mathrm{~g}$. The supernatant was discarded, and the DNA was washed with $600 \mu \mathrm{L} 70 \%$ ethanol and air dried on a clean bench. The DNA was dissolved in $150 \mu \mathrm{L} 0.1 \mathrm{X}$ TE buffer $(1 \mathrm{mM}$ Tris- $\mathrm{HCl}, \mathrm{pH} 8.0 ; 0.1 \mathrm{mM}$ EDTA, pH 8.0). The DNA concentration was determined using a NanoDrop 2000 spectrophotometer (Thermo Scientific, Wilmington, DE, USA) according to the manufacturer protocol.

\section{Real-time PCR primers and probe design}

The primers and fluorochrome-labeled probe were designed using the Primer Express 
3.0 software (Life Technologies, Carlsbad, CA, USA) based on the 2852-bp rDNA sequences of $U$. virens (GenBank accession No. AB105954). This rDNA region, which is a unique region in the $U$. virens genome and is not present in any of the other organisms, allowed for the design of specific primers and a probe for real-time PCR (Ashizawa et al., 2010). The primers and probe were synthesized (Life Technologies), and their sequences were shown in Table 1. The probe was labeled at the $5^{\prime}$ terminus with fluorescein (FAM) and near the $3^{\prime}$ terminus with a dark quencher dye (TAMRA). The primer pair amplified a product that was $84 \mathrm{bp}$ in length.

Table 1. Oligonucleotide primers and TaqMan probe sequence used for real-time PCR detection.

\begin{tabular}{ll}
\hline Primer or probe & Sequence (5' to $\left.3^{\prime}\right)$ \\
\hline RFS-F & GCGAGAGGATGCTACAATGGA \\
RFS-R & AATCCGCAGCCAAGCCCCACG \\
Probe & GCATACGGGGAAGGTTCAGA \\
\hline
\end{tabular}

\section{Standard curves and real-time PCR conditions}

To obtain the real-time PCR detection limits for $U$. virens DNA, a standard curve based on the threshold cycle $\left(\mathrm{C}_{\mathrm{T}}\right)$ for a dilution series of pure $U$. virens DNA $\left(1,0.5,1 \times 10^{-1}\right.$, $1 \times 10^{-2}, 1 \times 10^{-3}$, and $1 \times 10^{-4} \mathrm{ng} / \mu \mathrm{L}$, diluted with $0.1 \mathrm{X}$ TE buffer) was constructed and triplicate real-time PCR was performed. In order to quantitatively detect $U$. virens DNA in the actual rice samples, another standard curve based on the $\mathrm{C}_{\mathrm{T}}$ for the same dilution series of $U$. virens DNA (pure $U$. virens DNA diluted with $20 \mathrm{ng} / \mu \mathrm{L}$ negative-control rice spikelet DNA solution) was also constructed. The negative control rice samples were planted in the sterile culture room.

Real-time PCR was performed on an Applied Biosystems 7500 Real Time PCR System (Life Technologies). The amplification was carried out in a total volume of $25 \mu \mathrm{L}$, which contained $500 \mathrm{nM}$ each primer, $200 \mathrm{nM}$ probe, $12.5 \mu \mathrm{L}$ ABGene Absolute QPCR Rox Mix (Thermo Scientific), $5 \mu \mathrm{L}$ DNA template (different dilution samples), and nuclease-free water. The amplification was carried out using the following program: $5 \mathrm{~min}$ at $95^{\circ} \mathrm{C}$ for pre-denaturation and then 50 cycles of $10 \mathrm{~s}$ at $95^{\circ} \mathrm{C}$ and $30 \mathrm{~s}$ at $60^{\circ} \mathrm{C}$. The $\mathrm{C}_{\mathrm{T}}$ values were automatically calculated with the 7500 software version 2.0.5 (Life Technologies). The standard curves were obtained by plotting the $\mathrm{C}_{\mathrm{T}}$ value versus the logarithm of the concentration of each dilution of $U$. virens DNA.

\section{Quantitative detection of different samples by real-time PCR}

DNA from different artificially infected rice plants or field rice plants were extracted and analyzed by real-time PCR using the primer pair and probe. The DNA concentration of each rice sample was diluted to $20 \mathrm{ng} / \mu \mathrm{L}$ with $0.1 \mathrm{X}$ TE buffer. The quantity of $U$. virens DNA in a rice sample was calculated using the standard curve. For comparative purposes, the DNA samples were also analyzed by a nested-PCR assay according to the method of Zhou et al. (2003).

\section{Comparison of the real-time PCR assay and the pathogenicity test}

In order to compare the results of the real-time PCR assay and the pathogenicity test 
of $U$. virens, artificially infected rice plants were also infected by the dilution series of the conidial suspension $\left(10^{5}, 10^{6}, 10^{7}\right.$, and $10^{8}$ conidia $\left./ \mathrm{mL}\right)$. At least 10 panicles were inoculated for each treatment. Fifty days after the artificial injection, the number of smut balls per panicle was counted. Rice panicle samples from all treatments were harvested and mixed. Then, DNA was extracted and analyzed by real-time PCR by using the primer pair and probe.

\section{RESULTS}

\section{DNA extraction}

The DNA quality is critical for reproducible results in the real-time PCR assay (Cankar et al., 2006). U. virens and rice have a different cell structure. Different DNA extraction methods were used in many studies (Ashizawa et al., 2010; Nejat et al., 2010). However, different DNA extraction methods would influence the quantitative results of the real-time PCR (Tilburg et al., 2010). In this study, U. virens and rice genomic DNA were extracted individually or together by the same extraction method. Typically, DNA samples have an $\mathrm{A}_{260} / \mathrm{A}_{280}$ ratio $>1.8$ and $\mathrm{A}_{260} / \mathrm{A}_{230}$ ratio $>2.0$ (Table 2), which suggests that the DNA quality is sufficient to further examination. The $\mathrm{A}_{260} / \mathrm{A}_{280}$ ratio ranged from 1.92 to 2.06, and the $\mathrm{A}_{260} /$ $\mathrm{A}_{230}$ ratio ranged from 2.03 to 2.14 (Table 2). These results indicated that the DNA had little contamination of proteins, polysaccharides, and polyphenols. These contaminants, especially polyphenols, would inhibit the enzymatic activity of the DNA polymerase and affect the PCR amplification efficiency (Li et al., 2013).

Table 2. Concentrations, purities of DNA of Ustilaginoidea virens and the representative rice samples DNA.

\begin{tabular}{lccr}
\hline Samples & DNA concentration $(\mathrm{ng} / \mu \mathrm{L})$ & $\mathrm{A}_{260} / \mathrm{A}_{280}$ & $\mathrm{~A}_{260} / \mathrm{A}_{230}$ \\
\hline$U$. virens & $39.08 \pm 7.51$ & $1.95 \pm 0.06$ & $2.04 \pm 0.07$ \\
Rice- $T_{0}$ & $83.08 \pm 9.13$ & $2.06 \pm 0.05$ & $2.14 \pm 0.05$ \\
Rice- $T_{1}$ & $77.55 \pm 8.43$ & $1.99 \pm 0.03$ & $2.09 \pm 0.05$ \\
Rice- $T_{2}$ & $75.32 \pm 6.33$ & $1.95 \pm 0.06$ & $2.05 \pm 0.04$ \\
Rice-T & $71.80 \pm 5.22$ & $1.98 \pm 0.04$ & $2.08 \pm 0.08$ \\
Rice- $T_{4}$ & $65.28 \pm 7.86$ & $1.92 \pm 0.07$ & $2.03 \pm 0.06$ \\
\hline
\end{tabular}

Data are reported as means $\pm \mathrm{SD}, \mathrm{N}=8 ;$ Rice- $\mathrm{T}_{0}=$ rice samples sampled before artificial injection; Rice- $\mathrm{T}_{1}=$ rice samples sampled after artificial injection 10 days; Rice- $\mathrm{T}_{2}=$ rice samples sampled after artificial injection 20 days; Rice- $\mathrm{T}_{3}=$ rice samples sampled after artificial injection 30 days; Rice- $\mathrm{T}_{4}=$ rice samples sampled after artificial injection 40 days.

\section{Standard curves for real-time PCR}

In order to quantitatively detect $U$. virens DNA in the actual rice samples, the standard curves were generated using $U$. virens DNA concentrations that ranged from $0.1 \mathrm{pg} / \mu \mathrm{L}$ to 1 $\mathrm{ng} / \mu \mathrm{L}$ by 2 different dilution methods. The real-time PCR detection limit for $U$. virens DNA was $0.1 \mathrm{pg} / \mu \mathrm{L}\left(1 \times 10^{-4} \mathrm{ng} / \mu \mathrm{L}\right)$ (Figure 1). The curves of the 2 different dilution methods are described by $\mathrm{y}=-3.3692 \mathrm{x}+19.583\left(y\right.$ is the $\mathrm{C}_{\mathrm{T}}$, and $x$ is the logarithm of the starting $U$. virens DNA concentration) and $\mathrm{y}=-3.2341 \mathrm{x}+20.515$. The $\mathrm{R}^{2}$ value (coefficient of determination) was 1 for the samples diluted with 0.1X TE buffer and 0.9999 for the samples diluted with a negative-control rice spikelet DNA solution. The $U$. virens DNA concentration and $\mathrm{C}_{\mathrm{T}}$ were highly correlated for the primer pair and probe. 
Standard curves have been constructed in other studies by using pure pathogen DNA or recombinant plasmid DNA diluted with water (Ashizawa et al., 2010; Nejat et al., 2010). Because the components of the practical rice samples of DNA are different from those of the pure pathogen DNA or recombinant plasmid DNA samples, using these artificial standard curves to quantify the amount of DNA in the actual samples might result in deviations between the real-time PCR assay results and the actual amount. We observed that the negative-control rice spikelet DNA dilution method provided a higher $\mathrm{C}_{\mathrm{T}}$ value than the $0.1 \mathrm{X}$ TE dilution method at the same $U$. virens DNA concentration (Figure 1), which suggested that PCR replication was affected by the other components that were in the practical samples. Therefore, the results would be more accurate using a standard curve based on DNA samples that were diluted with rice genomic DNA.

\section{(A)}

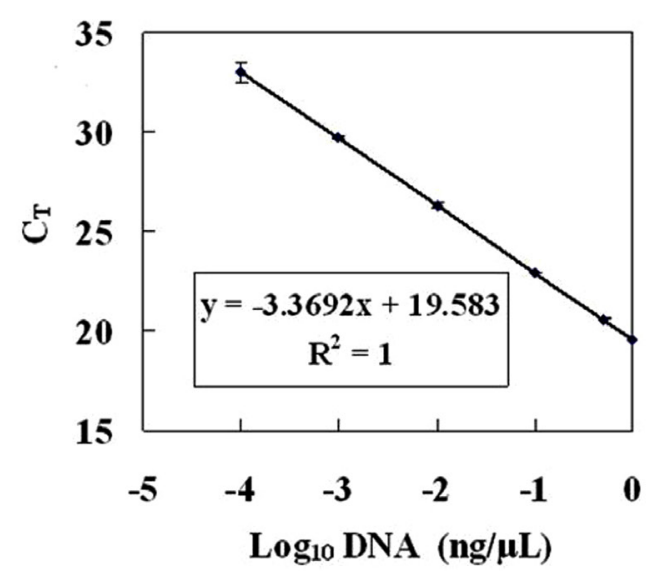

(B)

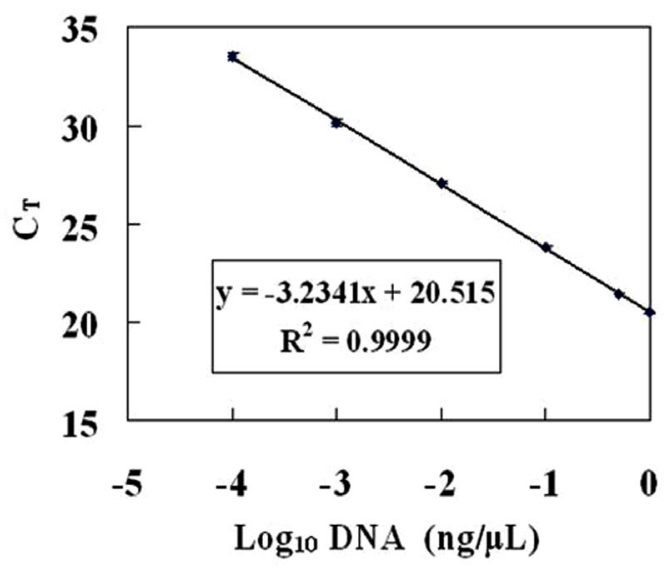

Figure 1. Standard curve for Ustilaginoidea virens DNA in real-time PCR assay. (A). Pure U. virens DNA diluted by $0.1 \mathrm{X}$ TE buffer. (B). Pure $U$. virens DNA diluted by $20 \mathrm{ng} / \mu \mathrm{L}$ negative control rice spikelets DNA solution. (Each point represents the mean of three replicates. Error bars represent the standard deviation.).

\section{Quantitative of $U$. virens by real-time PCR}

The mean $\mathrm{C}_{\mathrm{T}}$ and quantity of $U$. virens $\mathrm{DNA}$ from different artificially infected rice plants or field rice plants are shown in Table 3. The $\mathrm{C}_{\mathrm{T}}$ values obtained from different artificially infected rice plants or field rice plants ranged from 26.76 to 34.79 , whereas the negative control had $\mathrm{C}_{\mathrm{T}}$ values above 38 (Table 3 ). In the real-time PCR and nested-PCR assays, the detection limits of $U$. virens DNA were $40 \mathrm{fg}\left(3.85 \times 10^{-5} \mathrm{ng} / \mu \mathrm{L}\right)$ and $850 \mathrm{fg}\left(8.53 \times 10^{-4} \mathrm{ng} /\right.$ $\mu \mathrm{L}$ ), respectively (Table 3 ). Overall, the real-time PCR assay showed significantly higher sensitivity for detecting the $U$. virens DNA than the conventional nested-PCR assay.

\section{Comparison of the real-time PCR assay and the pathogenicity test}

The mean $\mathrm{C}_{\mathrm{T}}$ values and smut balls per panicle from the rice plants infected by dif- 
ferent concentrations of a conidia suspension are shown in Table 4. The concentrations of the conidia suspension ranged from $10^{5}$ to $10^{8}$ conidia $/ \mathrm{mL}$, the relevant $\mathrm{C}_{\mathrm{T}}$ values ranged from 27.48 to 23.62 , and the average number of smut balls per panicle ranged from 2.5 to 8.6 (Table 4). In other words, the $U$. virens DNA concentration obtained from the real-time PCR assay was consistent with the average number of smut balls per panicle.

Table 3. Real-time PCR and nested-PCR results for the representative samples.

\begin{tabular}{lcccc}
\hline Samples & Replicates & Mean $\mathrm{C}_{\mathrm{T}}$ & Nested-PCR & U. virens DNA concentration $(\mathrm{ng} / \mu \mathrm{L})^{*}$ \\
\hline Rice-T $_{0}$ & 3 & 37.34 & - & - \\
Rice-T $_{1}$ & 3 & 34.79 & - & $3.85 \times 10^{-5}$ \\
Rice-T $_{2}$ & 3 & 32.55 & - & $1.90 \times 10^{-4}$ \\
Rice-T $_{3}$ & 3 & 30.44 & + & $8.53 \times 10^{-4}$ \\
Rice-T & 3 & 28.21 & + & $4.18 \times 10^{-3}$ \\
Rice-SY & 3 & 37.55 & - & - \\
Rice-HF & 3 & 29.43 & + & $1.75 \times 10^{-3}$ \\
Rice-JZ & 3 & 26.76 & + & $1.17 \times 10^{-2}$ \\
Positive control & 3 & 20.51 & - & 1.0 \\
Negative control & 3 & 38.62 & +
\end{tabular}

The symbol (+) means where the product was detected, and the symbol (-) means no product detected. ${ }^{*} \mathrm{DNA}$ concentration calculated from the standard curve (Figure 1B) and is based on the average of 3 independent estimates for each replicate. Rice- $\mathrm{T}_{0}=$ rice samples sampled before artificial injection; Rice- $\mathrm{T}_{1}=$ rice samples sampled after artificial injection 10 days; Rice- $\mathrm{T}_{2}=$ rice samples sampled after artificial injection 20 days; Rice- $\mathrm{T}_{3}=$ rice samples sampled after artificial injection 30 days; Rice- $\mathrm{T}_{4}=$ rice samples sampled after artificial injection 40 days; Rice$\mathrm{SY}=$ rice samples sampled from Sanya of Hainan province (RFS non-epidemic area); Rice-HF = rice samples sampled from Hefei of Anhui Province (RFS epidemic area); Rice-JZ = rice samples sampled from Jinzhai of Anhui Province (RFS outbreak area); Positive control = pure $U$. virens DNA diluted by $20 \mathrm{ng} / \mu \mathrm{L}$ negative control rice spikelets DNA; Negative control = negative control rice spikelets DNA.

Table 4. Comparison of real-time PCR assay and pathogenicity test of Ustilaginoidea virens.
\begin{tabular}{lccc}
\hline Concentration of conidia suspension & Mean $\mathrm{C}_{\mathrm{T}}$ & $\begin{array}{c}\text { U. virens } \mathrm{DNA} \\
\text { concentration }(\mathrm{ng} / \mu \mathrm{L})^{*}\end{array}$ & $\begin{array}{c}\text { Average number of } \\
\text { smut balls per panicle }\end{array}$ \\
\hline $10^{5}$ conidia $/ \mathrm{mL}$ & 27.48 & $7.02 \times 10^{-3}$ & 2.5 \\
$10^{6}$ conidia $/ \mathrm{mL}$ & 26.29 & $1.64 \times 10^{-2}$ & 3.2 \\
$10^{7}$ conidia $/ \mathrm{mL}$ & 25.05 & $3.96 \times 10^{-2}$ & 5.3 \\
$10^{8}$ conidia $/ \mathrm{mL}$ & 23.62 & $1.10 \times 10^{-1}$ & 8.6 \\
\hline
\end{tabular}

*DNA concentration calculated from the standard curve (Figure 1B) and is based on the average of 3 independent estimates for each replicate.

\section{DISCUSSION}

The reliability of real-time PCR could be influenced by many factors, such as DNA quality, primers, and the probe (Bustin et al., 2009). Different DNA extraction methods could influence the DNA quality, which could affect PCR amplification (Tilburg et al., 2010). In order to obtain reproducible results and eliminate the influence of the DNA extraction method (Cankar et al., 2006), we used the same extraction protocol for DNA isolation from $U$. virens and rice samples. The standard curve of the DNA dilutions showed linearity $\left(\mathrm{R}^{2}=0.9999\right)$ in the real-time PCR assay; this result indicated that the quality of the DNA was suitable for the real-time PCR assay. The primer pair and probe in this study showed better detection results than the universal probe from the universal probe library (data not shown) (Ashizawa et al., 
2010). Moreover, the primers that amplified short DNA fragments showed better results than those that amplified long DNA fragments in the real-time PCR assay (Tilburg et al., 2010). The primer pair in this study amplified a product that was 84 bp in length. Taken together, the PCR system that we established is reliable and sensitive.

Relative and absolute quantification are widely used strategies to estimate DNA concentrations from $\mathrm{C}_{\mathrm{T}}$ values. Absolute quantification requires the construction of a standard curve, which involves the amplification of known amounts of target DNA. Plasmid DNA is often used to construct a standard curve because of its high quality and purity. For absolute quantification, the assumption that plasmid DNA and target genomic DNA amplify with the same efficiency must be made (Sivaganesan et al., 2008). In fact, many factors such as DNA stability, complexity, and secondary structure could significantly affect the amplification (Sivaganesan et al., 2008). Therefore, using the standard curve constructed with plasmid DNA would affect the quantification target DNA template that is complex genomic DNA instead of plasmid DNA. Moreover, a standard curve of genomic DNA isolated from actual rice samples is better fit to quantify the DNA amount than a standard curve of DNA isolated from pure cultures (Martín et al., 2006). In this study, U. virens DNA was diluted using a solution of the negative control rice spikelets DNA to construct the standard curve. It is fit to quantify the amount of $U$. virens DNA in the actual samples.

The conventional nested-PCR assay has been used to detect $U$. virens in rice (Zhou et al., 2003). The nested-PCR assay is less sensitive than the real-time PCR assay and cannot be used to quantify the amount of target DNA; therefore, it can only be used to indicate the presence or absence of the target DNA. The real-time PCR assay has the advantage of calculating the absolute quantity of starting target DNA within $2 \mathrm{~h}$. The real-time PCR assay has been employed for universal phytopathogen detection based on the 16S ribosomal RNA (rRNA) gene and 23S rRNA gene (Nejat et al., 2010). Ashizawa et al. (2010) developed a real-time PCR assay to clarify the relationship between the false smut occurrence on rice and the quantification of $U$. virens from soil in Japan. As a result, the real-time PCR technology provides new opportunities to detect and study $U$. virens and other plant phytopathogens.

In conclusion, the method for quantifying $U$. virens DNA in this study is more sensitive and reproducible than the conventional nested-PCR assay, in both artificially and naturally infected rice samples. The $U$. virens DNA concentration obtained from the real-time PCR assay was consistent with the average number of smut balls per panicle. The real-time PCR assay will be a useful tool for rapidly diagnosing and monitoring $U$. virens. Additionally, it would be beneficial with respect to breeding the RFS-resistant rice variety.

\section{ACKNOWLEDGMENTS}

Research supported by the National Key Technology R\&D Program of China (grant \#2012BAD07B01), Youth Foundation of President of the Anhui Agricultural Academy of Sciences (\#13B0101, \#13B0102, \#13B0113, \#13B0114), and Creative Team Foundation of the Anhui Agricultural Academy of Sciences (\#13C0101).

\section{REFERENCES}

Ashizawa T, Takahashi M, Moriwaki J and Hirayae K (2010). Quantification of the rice false smut pathogen Ustilaginoidea virens from soil in Japan using real-time PCR. Eur. J. Plant Pathol. 128: 221-232. 
Bustin SA, Benes V, Garson JA, Hellemans J, et al. (2009). The MIQE guidelines: minimum information for publication of quantitative real-time PCR experiments. Clin. Chem. 55: 611-622.

Cankar K, Štebih D, Dreo T and Žel J (2006). Critical points of DNA quantification by real-time PCR-effects of DNA extraction method and sample matrix on quantification of genetically modified organisms. BMC Biotechnol. 6: 37.

Ginzinger DG (2002). Gene quantification using real-time quantitative PCR: an emerging technology hits the mainstream. Exp. Hematol. 30: 503-512.

Harrison NA, Womack M and Carpio ML (2002). Detection and characterization of a lethal yellowing (16SrIV) group phytoplasma in Canary Island date palms affected by lethal decline in Texas. Plant Dis. 86: 676-681.

Ji HP and Zheng HJ (1995). Rice false smut in China. Helongjiang. Agr. Sci. 5: 40-41.

Koiso Y, Li Y, Iwasaki S, Hanaoka K, et al. (1994). Ustiloxins, antimitotic cyclic peptides from false smut balls on rice panicles caused by Ustilaginoidea virens. J. Antibiot. 47: 765-773.

Li H, Li J, Cong XH, Duan YB, et al. (2013). A high-throughput, high-quality plant genomic DNA extraction protocol. Genet. Mol. Res. 12: 4526-4539.

Lu DH, Yang XQ, Mao JH, Ye HL, et al. (2009). Characterising the pathogenicity diversity of Ustilaginoidea virens in hybrid rice in China. J. Plant Pathol. 91: 443-451.

Maciel BM, Dias JC, Romano CC, Sriranganathan N, et al. (2011). Detection of Salmonella enteritidis in asymptomatic carrier animals: comparison of quantitative real-time PCR and bacteriological culture methods. Genet. Mol. Res. 10: 2578-2588.

Martín B, Jofre A, Garriga M, Pla M, et al. (2006). Rapid quantitative detection of Lactobacillus sakei in meat and fermented sausages by real-time PCR. Appl. Environ. Microbiol. 72: 6040-6048.

Nejat N, Sijam K, Abdullah SNA, Vadamalai G, et al. (2010). Development of a TaqMan real-time PCR for sensitive detection of the novel phytoplasma associated with coconut yellow decline in Malaysia. J. Plant Pathol. 92: 769-773.

Ou SH (1972). Rice Diseases, Commonwealth. Mycological Institute Kew, Surrey.

Sivaganesan M, Seifring S, Varma M, Haugland RA, et al. (2008). A Bayesian method for calculating real-time quantitative PCR calibration curves using absolute plasmid DNA standards. BMC Bioinformatics 9: 120.

Tanaka E and Tanaka C (2008). Phylogenetic study of clavicipitaceous fungi using acetaldehyde dehydrogenase gene sequences. Mycoscience 49: 115-125.

Tilburg JJ, Melchers WJ, Pettersson AM, Rossen JW, et al. (2010). Interlaboratory evaluation of different extraction and real-time PCR methods for detection of Coxiella burnetii DNA in serum. J. Clin. Microbiol. 48: 3923-3927.

Zhou YL, Izumitsu K, Sonoda R, Nakazaki T, et al. (2003). PCR-based specific detection of Ustilaginoidea virens and Ephelis japonica. J. Phytopathol. 151: 513-518. 